\title{
Effects of Bariatric Surgery on Weight Loss and Quality of Life
}

\author{
Signe Nielsen ${ }^{\star}$, Maria S. Svane, Kirstine N. Bojsen-Møller and Sten Madsbad
}

Department of Endocrinology, Hvidovre University Hospital, University of Copenhagen, Kettegaard Allé 30, DK-2650 Hvidovre, Denmark

*Corresponding author: Signe Nielsen, Bachelor of Medicine (MB), Department of Endocrinology, Hvidovre University Hospital, University of Copenhagen, Kettegaard Allé 30, DK-2650 Hvidovre, Denmark ; Phone: +45 38623032 ; Fax: +45 38626974 ; E-mail: signe.nielsen.04@regionh.dk

Received date: July 25, 2014, Accepted date: October 01, 2014, Published date: October 8, 2014

Copyright: (c) 2014 Signe Nielsen et al. This is an open-access article distributed under the terms of the Creative Commons Attribution License, which permits unrestricted use, distribution, and reproduction in any medium, provided the original author and source are credited.

\begin{abstract}
Bariatric surgery is the most effective treatment for severe obesity. The mean weight loss is approximately $30-40$ $\mathrm{kg} / 11-15 \mathrm{BMl}$ units or approximately $60 \%$ of excess body weight. Many patients pursue bariatric surgery for improvement in their physical image and self-esteem rather than somatic health improvement. Health-related quality of life improves after bariatric surgery and the long-term improvements are positively associated with the long-term amount of weight reduction. After the major weight loss, excess skin present problems for the patients and many patients want body contouring by plastic surgery. Plastic surgery adds further improvement in health-related quality of life.
\end{abstract}

Conclusion: Bariatric surgery leads to sustained weight loss and improved health-related quality of life. Plastic surgery has an additional positive effect on quality of life.

Keywords: Bariatric surgery; Weight loss; Health-related quality of life; Plastic surgery

\section{Introduction}

Obesity is a worldwide epidemic and is currently one of the greatest health problems. Obesity is associated with increased risk of serious health consequences as type 2 diabetes, cardiovascular disease, certain cancers and increased mortality [1].

Bariatric surgery - and Roux-en-Y gastric bypass (RYGB) in particular - is superior to both medical therapy and intensive lifestyle interventions with regard to achieve substantial and durable weight loss. The mean weight loss is approximately 30-40 kg/11-15 BMI units or approximately $60 \%$ of excess body weight after RYGB [2]. Bariatric surgery is thus the most effective therapy to obtain substantial, longlasting weight loss and furthermore improvement or remission of obesity related co-morbidities is seen. The beneficial effect of the operations on diabetes, hypertension and hyperlipidaemia is well described in the literature $[2,3]$ and bariatric surgery also reduces the overall mortality compared to non-operated control groups $[4,5]$. Besides these obvious advantages on physical health, bariatric surgery also influences psychological and health-related quality of life (HRQL). The well-being of the patients and their perceived quality of life postoperatively are important issues when discussing pros and cons of bariatric surgery.

The aim of this review is to provide an update on bariatric surgery and changes in quality of life, focusing especially on the three most common bariatric procedures, RYGB, sleeve gastrectomy (SG) and laparoscopic adjustable gastric banding (LAGB) (Figure 1).

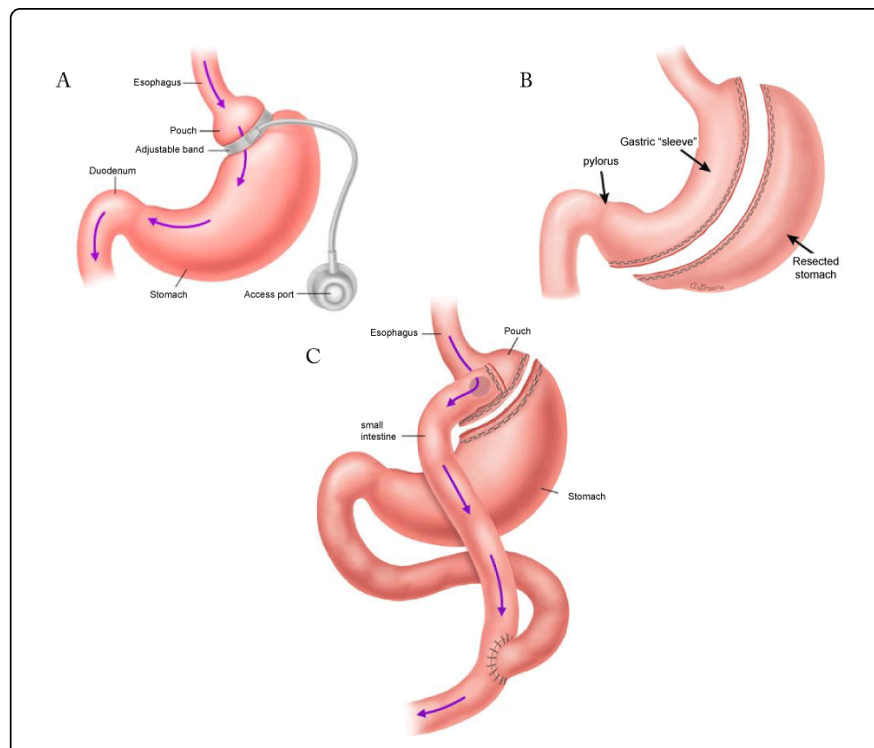

Figure 1: Bariatric procedures:(A) Laparoscopic adjustable gastric banding, (B) Sleeve gastrectomy and (C)Roux-en-Y gastric bypass procedure. Further information about the procedures is given in the text.

\section{Bariatric Surgical Procedures}

Bariatric procedures have traditionally been divided into restrictive procedures, including Laparoscopic adjustable gastric banding (LAGB), Vertical banded gastroplasty (VBG)and Sleeve gastrectomy $(S G)$,and the socalled "combined restrictive and malabsorptive" procedures involving bypass of segments of the gastrointestinal tract as Roux-en-Y gastric bypass (RYGB) and Biliopancreatic diversion (BPD) [6]. However, this classification may be outdated as the 
mechanisms of actions are more complex and seem to involve neural (LAGB) as well as gut hormone mediated signals (RYGB, SG) to appetite regulating centers of the brain [7]. RYGB is the most commonly performed bariatric procedure worldwide, followed by SG and LAGB, whereas BPD is used less often [8].

$L A G B$ is a procedure where a small silicone band is placed around the upper portion of the stomach creating an adjustable and reversible small stomach pouch $(30 \mathrm{~mL})$ above the band with a small opening through which food can enter the stomach. The band is lined by an inflatable cuff, which is linked to a subcutaneous abdominal reservoir, allowing adjustment of the pouch outflow by removing or adding saline (Figure 1) [6]. VBG creates a permanently small stomach pouch by using a band and staples. Recent research has indicated that it is distension of the small pouch activating gastric sensory receptors that induce satiety via vagal nerve signaling [7]. Another possible mechanism of action is induction of satiety by direct pressure or contact of the band with the gastric wall [7]. However, altered secretion of anorexigenic hormones do not seem to be involved in appetite regulation after LAGB or VBG [7,9].

$S G$ creates a narrow tubular pouch, like the shape of a banana, by removing a large portion (70-80\%) of the stomach following the major curve (Figure 1) [6]. Postoperatively, nutrient delivery to the small intestine is accelerated, which is likely to elicit an altered gut hormone response affecting appetite control and insulin release [7]. Exaggerated release of glucagon-like-peptide 1 (GLP-1) as well as other anorexigenic hormones are likely to explain the beneficial effects on weight as well as an early effect of type 2 diabetes [7].

$R Y G B$ involves creation of a stomach pouch of $30 \mathrm{~mL}$ around the oesophagus and division of the small intestine $50-75 \mathrm{~cm}$ distal to the ligament of Treitz (Figure 1). The pouch is connected to the distal part of the severed small intestine (the "alimentary limb"). The proximal part of the severed small intestine is reattached about $100 \mathrm{~cm}$ below the division (the "secretory limb"). Postoperatively, nutrients bypass the major part of the stomach, the duodenum and the upper part of the jejunum and are mixed with biliary and pancreatic secretions at the site of the entero-entero anastomosis. The weight loss is primarily explained by the postprandial changes in the levels of gut hormones, including GLP-1, gastric inhibitory polypeptide (GIP), Peptide YY (PYY), cholecystokinin (CCK) and ghrelin [6]. Weight loss and remission of type 2 diabetes are slightly superior after RYGB compared to SG in a randomized controlled trial (RCT) at three years follow-up [10]. The marked changes in postprandial hormone responses reported after SG and RYGB lead to a classification of the two procedures as "metabolic surgery".

$B P D$ with duodenal switch reduces the stomach to a tubular pouch, similar to SG. The distal part of the small intestine is connected to the pouch bypassing a larger portion of the small intestine than the gastric bypass operation [6]. In contrary to RYGB [11], malabsorption is a significant contributor to the beneficial effect on weight and type 2 diabetes after BPD probably explaining why weight loss is larger and more durable than with the other procedures [12]. However, nutritional deficiencies are also more common after BPD compared to other bariatric procedures [13].

\section{Weight loss after bariatric surgery}

Weight loss in severely obese patients after bariatric procedures is best described by the percentage of excess body weight loss (\%EBWL) or BMI loss (\%EBMIL) calculated by the formula (preoperative weight or BMI - postoperative weight or BMI) / (preoperative weight or BMI - ideal body weight or 25$) \times 100 \%$.

In a meta-analysis by Buchwald et al. \%EBWL was greatest in the BPD subgroup (64\%), followed by RYGB (60\%), VBG (56\%) and LAGB (46\%) [2]. The weight loss after SG seems to be greater than weight loss after LAGB and VBG but slightly lower than after RYGB [10].

The Swedish Obese Subject (SOS) Study is a major prospective, non-randomized intervention study comparing three types of bariatric surgery $(n=2010$, fixed or adjustable banding $(n=376)$, VBG $(n=1369)$ and RYGB $(\mathrm{n}=265)$ ) with nonsurgical treated patients undergoing conventional treatment for obesity $(n=2037)$. The weight changes in the surgical subgroups over 20 years were consistent with the results of Buchwald et al. and the nonsurgical treated patients showed the smallest weight changes during the follow-up (Figure 2). The phases of weight changes in the surgical subgroups indicated maximal weight loss after 1-2 years, weight regains in the following years followed by stable weights after 8-10 years (Figure 2) [14]. Approximately $5-10 \%$ of patients regain the weight loss after RYGB and even a higher number of patients after LAGB. On average it is expected that $20-25 \%$ of the weight lost will be regained over a period of 10 years after bariatric surgery [15].

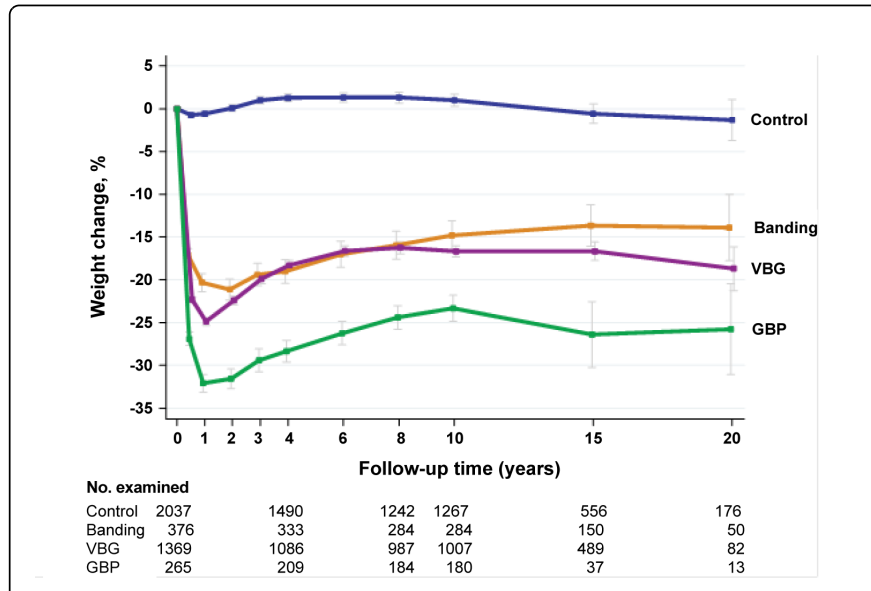

Figure 2: Mean percentage weight change from baseline over 20 years of follow-up in the Swedish Obese Subjects Study [14]. Control $=$ controls receiving conventional obesity treatment. Banding = laparoscopic adjustable gastric banding. $\mathrm{VBG}=$ vertical banded gastroplasty. GBP = gastric bypass.

\section{Risks of Bariatric Surgery}

Concerns about the safety of bariatric surgery have grown with its increasing popularity. Perioperative mortality $(30$ days $)$ is approximately $0.1 \%$ for LAGB and VBG and $0.3 \%$ after RYGB $[16,17]$. In general, higher rates of acute and long term complications have been reported among patients undergoing the more invasive procedures (BPD, RYGB and SG) compared to banding procedures [17].

Acute complications after bariatric surgery include bleeding, infections, anastomotic leaks, arrhythmias and pulmonary emboli. Postoperative long-term complications are nutritional deficiencies, internal hernias and anastomotic stenosis. To avoid postoperative 
nutritional deficiencies, especially after BPD, RYGB and SG, patients must supplement lifelong with tablets of calcium, D-vitamin, Avitamin, iron, folate, thiamine and injections of B12-vitamin [18]. Anemia due to iron deficiency is one of the major concerns after BPD and RYGB but may also be seen after SG, especially in premenopausal women [13]. Postoperative monitoring is of major importance as all patients may not respond adequately to tablet supplementation and may require intravenous iron supplementation. Adequate protein intake is emphasized postoperatively, especially after BPD. Ideally 60-120 gram/day or $1 \mathrm{gram} / \mathrm{kg}$ is essential for preservation of lean body mass and wound healing. Protein deficiency is most commonly seen one to two years after surgery and is confirmed by a low albumin level $[13,18]$.

After RYGB and to a lesser degree after SG some patients will experience dumping symptoms including abdominal pain/cramping, sweating, tachycardia and diarrhea beginning 30 to 60 minutes after eating. A few patients develop postprandial hypoglycemia due to postprandial hyperinsulinemia accompanying an exaggerated GLP-1 response [18]. Postprandial hypoglycemia is thus not observed after LAGB and VBG.

Although, safety is of major importance when choosing among the different bariatric procedures, it is not the only relevant metric. Effectiveness and durability on weight loss and improvements of comorbidities are critical [16]. Moreover, many obese individuals suffer from impaired HRQL and other forms of psychosocial distress including low self-esteem and may pursue bariatric surgery in order to improve quality of life, physical image and self-esteem $[19,20]$. Changes in HRQL after bariatric surgery are therefore important to consider.

\section{HRQL after bariatric surgery}

Many studies assessing the effects of bariatric surgery on HRQL suffer from methodological limitations such as small sample sizes, lack of control groups, short-term follow-up and retrospective designs [2128]. Five RCTs have compared bariatric surgery to other interventions $[10,12,29-31]$ and only two reported HRQL outcomes [10,30]. Recently Schauer et al. reported 3-year outcomes after randomization of 150 obese patients with uncontrolled type 2 diabetes to intensive medical therapy alone or medical therapy plus RYGB or SG. HRQL was significantly improved in the two surgical groups compared to the medical-therapy group [10], supplementary material. RYGB was slightly although not significantly superior to SG in improving HRQL. Furthermore, the greatest weight loss from baseline was reported in the RYGB group [10]. Another study found similar weight loss and improvements in HRQL among patients randomized to either SG or RYGB but did not include a non-surgical control group [32]. O'Brien et al. investigated changes in HRQL in patients randomized to LAGB or intensive lifestyle modifications [30]. After 2-years of follow-up LAGB was superior in reducing weight and improving HRQL. These results are consistent with longitudinal LAGB studies, which however did not include control groups [33-35].

The SOS-study evaluated HRQL after 10 years of follow-up in 655 surgically (LABG $(\mathrm{n}=161)$, VBG $(\mathrm{n}=457)$ and RYGB $(\mathrm{n}=37))$ versus 621 conventionally treated obese patients [36]. Although not randomized, surgically treated patients were closely matched on 18 parameters (gender, age, weight, height, waist and hip circumferences, systolic blood pressure, serum cholesterol and triglyceride levels, smoking status, diabetes, menopausal status and six psychological parameters) to the obese conventionally treated group and therefore this study provides the best long-term evidence at present for comparing bariatric surgery with conventional treatment for obesity. The overall changes in HRQL were closely linked to the amount of weight loss. HRQL in the surgical group improved during the first year of weight loss and peaked at 0.5 and 1 year, between years 1 and 6 the weight regain was attended by a decline in HRQL, whereupon the HRQL as well as the weight stabilized from years 6 to 10. Finally, at 10years follow-up significant weight losses were seen in the surgical groups with no significant weight loss in the control group and the HRQL in the surgical subgroup remained improved compared to baseline [36]. However, because RYGB is the most common bariatric procedure at present, the SOS study is limited by the small number of RYGB operated patients [14].

The Utah Obesity Study is a prospective cohort study of RYGB patients $(n=323)$ that included two control cohorts: individuals, who sought but did not have RYGB $(n=257)$ and severely obese subjects $(n=272)$ identified from a database [37]. HRQL was estimated at 2 and 6 years of follow-up $[38,39]$. RYGB operated patients had significantly greater weight loss and improvements in HRQL compared to the two control groups at 2-years follow-up [38]. At 6-years follow up, HRQL deteriorated slightly from the 2-year assessment which potentially could be explained by weight regain. However, HRQL improvements remained significantly larger in RYGB operated patients compared to both control groups [37,39].

Overall, bariatric surgery, RYGB in particular, causes a significant weight loss compared to non-surgical weight loss techniques which result in significant improvements in HRQL postoperatively.

\section{Plastic surgery and HRQL}

Excessive weight loss after bariatric surgery often leads to the perception of excess skin as a negative consequence of surgery motivating patients to seek plastic surgeons for skin removal and body contouring [40,41]. Excess skin is most pronounced in patients with preoperatively morbid obesity and greatest weight loss and with increasing age at the time of surgery. Common problems are abdominal skin folds (hanging abdominal pannus) and skin fold at the buttocks which can lead to discomfort of sitting. Excess skin at upper arms, thighs and the breast area can also cause trouble and skin infections can develop leading to erythema and unpleasant odours. Studies have indicated that $74-85 \%$ of RYGB patients want body contouring but that the majority $(80 \%)$ do not have surgery, often for economically reasons $[42,43]$.

A case-control study measured the impact of plastic surgery on HRQL by firstly comparing HRQL before and after plastic surgery and secondly by comparing HRQL between a subgroup of patients undergoing RYGB followed by plastic surgery and a matched subgroup undergoing RYGB alone [44]. The study confirmed previous findings of improved HRQL after RYGB which was directly related to weight loss. Moreover, the study showed that plastic surgery further improved HRQL in comparison to RYGB alone. Another report, from the same researchers, showed that patients who received plastic surgery after RYGB displayed a better long-term weight control than patients who had RYGB alone despite matching of preoperative BMI, sex and age and BMI and \%EBWL at the 2-year follow-up [45]. Another study showed sustained improvements in HRQL up to 7 years after RYGB followed by plastic surgery but did not include control groups [46]. 
In summary, bariatric surgery followed by plastic surgery seems to improve HRQL to a larger extent that bariatric surgery alone and may also contribute to better weight loss maintenance.

\section{Comment}

Obesity is an increasing cause of morbidity and mortality and at present bariatric surgery is the only intervention demonstrating substantial and sustained weight loss. Safety of the different procedures is an important consideration along with effects on comorbidities to obesity. However, quality of life after bariatric surgery is also an important clinical outcome. Therefore, the aim of this review was to provide an overview of changes in quality of life after different bariatric surgical procedures.

In general, long-term improvements in HRQL are positive associated with the degree of long-term weight reduction. RYGB is superior to the restrictive operations with respect to the amount and durability of weight loss and therefore long-term improvements in HRQL seem to be more favorable after gastric bypass. This is supported by an observational cohort study including 2949 SG patients and equal numbers of RYGB and LAGB patients. By comparing HRQL after SG, RYGB and LAGB, they found better HRQL after RYGB and SG than after LAGB [47]. However, a prospective randomized trial comparing RYGB with LAGB demonstrated that postoperative improvements in HRQL were larger in the RYGB group than in the LAGB group at 1-month follow-up but 1 year after surgery the HRQL did not differ between the two procedures [48]. Other non-randomized studies comparing LAGB and RYGB reached similar conclusions $[22,49,50]$.

In conclusion, bariatric surgery is superior to other interventions in achieving long-term sustained weight loss in morbidly obese patients leading to improvements of obesity-related comorbidities as well as marked improvements in HRQL. Changes in HRQL are closely related to weight loss and weight regain. Furthermore, plastic surgery has additional beneficial effects on HRQL and should be considered as a postoperative support to stabilize the long-term success of bariatric surgery.

\section{Acknowledgement:}

The review is not funded by any external sources.

\section{References}

1. Picot J, Jones J, Colquitt JL, Gospodarevskaya E, Loveman E, et al. (2009) The clinical effectiveness and cost-effectiveness of bariatric (weight loss) surgery for obesity: a systematic review and economic evaluation. Health Technol Assess 13: 1-190, 215-357.

2. Buchwald H, Estok R, Fahrbach K, Banel D, Jensen MD, et al. (2009) Weight and type 2 diabetes after bariatric surgery: systematic review and meta-analysis. Am J Med 122: 248-256.

3. Gloy VL, Briel M, Bhatt DL, Kashyap SR, Schauer PR, et al. (2013) Bariatric surgery versus non-surgical treatment for obesity: a systematic review and meta-analysis of randomised controlled trials. BMJ 347: 5934.

4. Sjöström L, Narbro K, Sjöström CD, Karason K, Larsson B, et al. (2007) Effects of bariatric surgery on mortality in Swedish obese subjects. N Engl J Med. 357: 741-52.

5. Adams TD, Gress RE, Smith SC, Halverson RC, Simper SC, et al. (2007) Long-term mortality after gastric bypass surgery. N Engl J Med 357: $753-$ 61.

6. Ashrafian H, Bueter M, Ahmed K, Suliman A, Bloom SR, et al. (2010) Metabolic surgery: an evolution through bariatric animal models. Obes Rev 11:907-20.
7. Madsbad S, Dirksen C, Holst JJ (2014) Mechanisms of changes in glucose metabolism and bodyweight after bariatric surgery. The Lancet Diabetes Endocrinology 2:152-64.

8. Buchwald H, Oien DM (2013) Metabolic/bariatric surgery worldwide 2011. Obes Surg 23: 427-36.

9. Le Roux CW, Welbourn R, Werling M, Osborne A, Kokkinos A, et al. (2007) Gut hormones as mediators of appetite and weight loss after Roux-en-Y gastric bypass. Ann Surg 246: 780-5.

10. Schauer PR, Bhatt DL, Kirwan JP, Wolski K, Brethauer SA, et al. (2014) Bariatric Surgery versus Intensive Medical Therapy for Diabetes - 3-Year Outcomes. N Engl J Med 370: 2002-13.

11. Odstrcil EA, Martinez JG, Santa Ana CA, Xue B, Schneider RE, et al. (2010) The contribution of malabsorption to the reduction in net energy absorption after long-limb Roux-en-Y gastric bypass. Am J Clin Nutr 92: 704-13.

12. Mingrone G, Panunzi S, De Gaetano A, Guidone C, Iaconelli A, et al. (2012) Bariatric surgery versus conventional medical therapy for type 2 diabetes. N Engl J Med 366: 1577-85.

13. Bal BS, Finelli FC, Shope TR, Koch TR (2012) Nutritional deficiencies after bariatric surgery. Nat Rev Endocrinol 8: 544-56.

14. Sjöström L (2013) Review of the key results from the Swedish Obese Subjects (SOS) trial - a prospective controlled intervention study of bariatric surgery. J Intern Med 273: 219-34.

15. Sjöström L, Lindroos A-K, Peltonen M, Torgerson J, Bouchard C, et al. (2004) Lifestyle, diabetes, and cardiovascular risk factors 10 years after bariatric surgery. N Engl J Med 351: 2683-93.

16. Buchwald H, Avidor Y, Braunwald E, Jensen MD, Pories W, et al. (2004) Bariatric surgery: a systematic review and meta-analysis. JAMA 292: 1724-37.

17. Flum DR, Belle SH, King WC, Wahed AS, Berk P, et al. (2009) Perioperative safety in the longitudinal assessment of bariatric surgery. $\mathrm{N}$ Engl J Med 361: 445-54.

18. Heber D, Greenway FL, Kaplan LM, Livingston E, Salvador J, et al. (2010) Endocrine and nutritional management of the post-bariatric surgery patient: an Endocrine Society Clinical Practice Guideline. J Clin Endocrinol Metab 95: 4823-43.

19. Sullivan M, Karlsson J, Sjöström L, Taft C, Bjorntorp P (2001) Why Quality of Life Measures Should Be Used in the Treatment of Patients with Obesity. International Textbook of Obesity. John Wiley \& Sond Ltd 485-510.

20. Kolotkin RL, Meter K, Williams GR (2001) Quality of life and obesity. Obes Rev 2: 219-29.

21. Aftab H, Risstad H, Søvik TT, Bernklev T, Hewitt S, et al. (2014) Fiveyear outcome after gastric bypass for morbid obesity in a Norwegian cohort. Surg Obes Relat Dis 10:71-8.

22. Müller MK, Wenger C, Schiesser M, Clavien P-A, Weber M (2008) Quality of life after bariatric surgery - a comparative study of laparoscopic banding vs. bypass. Obes Surg 18: 1551-7.

23. Julia C, Ciangura C, Capuron L, Bouillot J-L, Basdevant A, et al. (2013) Quality of life after Roux-en-Y gastric bypass and changes in body mass index and obesity-related comorbidities. Diabetes Metab 39: 148-54.

24. Mar J, Karlsson J, Arrospide A, Mar B, Martínez de Aragón G, et al. (2013) Two-year changes in generic and obesity-specific quality of life after gastric bypass. Eat Weight Disord 18: 305-10.

25. Laurino Neto RM, Herbella FAM (2013) Changes in quality of life after short and long term follow-up of Roux-en-Y gastric bypass for morbid obesity. Arq Gastroenterol 50:186-90.

26. Zeller MH, Reiter-Purtill J, Ratcliff MB, Inge TH, Noll JG (2011) Twoyear trends in psychosocial functioning after adolescent Roux-en- $Y$ gastric bypass. Surg Obes Relat Dis 7:727-32.

27. Järvholm K, Olbers T, Marcus C, Mårild S, Gronowitz E, et al. (2012) Short-term psychological outcomes in severely obese adolescents after bariatric surgery. Obesity20:318-23.

28. Karlsen TI, Lund RS, Røislien J, Tonstad S, Natvig GK, et al. (2013) Health related quality of life after gastric bypass or intensive lifestyle 
Citation: Nielsen S, Svane MS, Bojsen-Møller KN, Madsbad S (2014) Effects of Bariatric Surgery on Weight Loss and Quality of Life.

intervention: a controlled clinical study. Health Qual Life Outcomes 11:17.

29. Ikramuddin S, Korner J, Lee W-J, Connett JE, Inabnet WB, et al. (2013) Roux-en-Y gastric bypass vs intensive medical management for the control of type 2 diabetes, hypertension, and hyperlipidemia: the Diabetes Surgery Study randomized clinical trial. JAMA 309:2240-9.

30. O'Brien PE, Dixon JB, Laurie C, Skinner S, Proietto J, et al. (2006) Treatment of mild to moderate obesity with laparoscopic adjustable gastric banding or an intensive medical program: a randomized trial. Ann Intern Med 144:625-33.

31. Dixon JB, O’Brien PE, Playfair J, Chapman L, Schachter LM, et al. (2008) Adjustable gastric banding and conventional therapy for type 2 diabetes: a randomized controlled trial. JAMA 299:316-23.

32. Peterli R, Borbély Y, Kern B, Gass M, Peters T, et al. (2013) Early results of the Swiss Multicentre Bypass or Sleeve Study (SM-BOSS): a prospective randomized trial comparing laparoscopic sleeve gastrectomy and Roux-en-Y gastric bypass. Ann Surg258:690-5.

33. O'Brien PE, Dixon JB, Brown W, Schachter LM, Chapman L, et al. (2002) The laparoscopic adjustable gastric band (Lap-Band): a prospective study of medium-term effects on weight, health and quality of life. Obes Surg 12:652-60.

34. Pilone V, Mozzi E, Schettino AM, Furbetta F, Di Maro A, et al. (2012) Improvement in health-related quality of life in first year after laparoscopic adjustable gastric banding. Surg Obes Relat Dis 8:260-8.

35. Helmiö M, Salminen P, Sintonen H, Ovaska J, Victorzon M(2011) A 5year prospective quality of life analysis following laparoscopic adjustable gastric banding for morbid obesity. Obes Surg 21: 1585-91.

36. Karlsson J, Taft C, Rydén A, Sjöström L, Sullivan M (2007) Ten-year trends in health-related quality of life after surgical and conventional treatment for severe obesity: the SOS intervention study. Int J Obes 31: 1248-61.

37. Adams TD, Davidson LE, Litwin SE, Kolotkin RL, LaMonte MJ, et al. (2012) Health benefits of gastric bypass surgery after 6 years. JAMA 308:1122-31

38. Kolotkin RL, Crosby RD, Gress RE, Hunt SC, Adams TD (2009) Twoyear changes in health-related quality of life in gastric bypass patients compared with severely obese controls. Surg Obes Relat Dis 5: 250-6.

39. Kolotkin RL, Davidson LE, Crosby RD, Hunt SC, Adams TD (2012) Sixyear changes in health-related quality of life in gastric bypass patients versus obese comparison groups. Surg Obes Relat Dis 8: 625-33.
40. Knol JA (1994) Management of the problem patient after bariatric surgery. Gastroenterol Clin North Am 23: 345-69.

41. Staalesen T, Fagevik Olsén M, Elander A (2013) Experience of excess skin and desire for body contouring surgery in post-bariatric patients. Obes Surg 23: 1632-44.

42. Kitzinger HB, Abayev S, Pittermann A, Karle B, Bohdjalian A, et al (2012) After massive weight loss: patients' expectations of body contouring surgery. Obes Surg 22: 544-8.

43. Gusenoff JA, Messing S, O'Malley W, Langstein HN (2008) Temporal and demographic factors influencing the desire for plastic surgery after gastric bypass surgery. Plast Reconstr Surg 121: 2120-6.

44. Modarressi A, Balagué N, Huber O, Chilcott M, Pittet-Cuénod B (2013) Plastic surgery after gastric bypass improves long-term quality of life. Obes Surg 23: 24-30.

45. Balagué N, Combescure C, Huber O, Pittet-Cuénod B, Modarressi A (2013) Plastic surgery improves long-term weight control after bariatric surgery. Plast Reconstr Surg 132:826-33.

46. Van der Beek ESJ, Geenen R, de Heer F a G, van der Molen ABM, van Ramshorst B (2012) Quality of life long-term after body contouring surgery following bariatric surgery: sustained improvement after 7 years. Plast Reconstr Surg 130:1133-9.

47. Carlin AM, Zeni TM, English WJ, Hawasli AA, Genaw JA, et al. (2013) The comparative effectiveness of sleeve gastrectomy, gastric bypass, and adjustable gastric banding procedures for the treatment of morbid obesity. Ann Surg 257:791-7.

48. Nguyen NT, Slone J, Nguyen X-MT, Hartman JS, Hoyt DB (2009) A prospective randomized trial of laparoscopic gastric bypass versus laparoscopic adjustable gastric banding for the treatment of morbid obesity: outcomes, quality of life, and costs. Ann Surg 250:631-41.

49. Campos GM, Rabl C, Roll GR, Peeva S, Prado K, et al. (2011) Better weight loss, resolution of diabetes, and quality of life for laparoscopic gastric bypass vs banding: results of a 2-cohort pair-matched study. Arch Surg 146:149-55.

50. Hell E, Miller K, Moorehead MK, Norman S (2000) Evaluation of health status and quality of life after bariatric surgery: comparison of standard Roux-en-Y gastric bypass, vertical banded gastroplasty and laparoscopic adjustable silicone gastric banding. Obes Surg 10:214-9. 